\title{
Epitope tagging
}

\author{
Bill Brizzard
}

BioTechniques 44:693-695 (25th Anniversary Issue, April 2008)

doi $10.2144 / 000112841$

\begin{abstract}
Epitope tagging is widely used in the characterization of newly discovered proteins. This review presents an overview of how the technique evolved and how it is being used today, with a focus on its use in the study of protein-protein interactions. In addition, the evolution of the technique for proteomic analyses is described.
\end{abstract}

\section{INTRODUCTION}

Epitope tagging is a technique in which a known epitope is fused to a recombinant protein by means of genetic engineering. By choosing an epitope for which an antibody is available, the technique makes it possible to detect proteins for which no antibody is available. This is especially useful for the characterization of newly discovered proteins and proteins of low immunogenicity. By selection of the appropriate epitope and antibody pair, it is possible to find a combination with properties that are suitable for the desired experimental application, such as Western blot analysis, immunoprecipitation, immunochemistry, and affinity purification.

\section{How It Started}

The advent of hybridoma technology made monoclonal antibodies available, thereby providing a reproducible source of antibody. However, the first commercially available epitope tags were originally designed for protein purification. Examples of these early commercial products include the FLAG, $6 \times$ His, and glutathione-S-transferase (GST) systems. The FLAG tagging system in its original version included the antiFLAG M1 monoclonal antibody with calcium-dependent binding. FLAGtagged proteins can be eluted from the M1 antibody with EDTA (1). Likewise, the $6 \times$ His tag is used for purification of recombinant proteins by means of metal chelate chromatography (2). Similarly, GST-tagged proteins can be purified using glutathione agarose (3). In addition to the commercial tags, the development of the other tags such as HA (4) and c-myc (5) were reported by academic groups. As research progressed and recombinant DNA technology evolved, the utility of epitope tags in the study of protein interaction was recognized. The result was rapid growth in the number of available tag and antibody pairs. For example, the anti-FLAG M2 monoclonal antibody was made available commercially (6) as were monoclonal antibodies for the $6 \times$ His tag (7), HA tag, and c-myc tag.

\section{Where It Stands}

There are now numerous types of tags with different features suited to diverse applications. The tags discussed in this review are listed in Table 1. For information on additional tags, see Reference 8. The most significant of these was the discovery of fluorescent protein reporters (9), which made it possible to detect proteins intracellularly without the need of a secondary reagent. The numerous applications of fluorescent reporters are beyond the scope of this review. While the use of epitope-tagging facilitates the study and characterization of newly discovered proteins, the technique does have some limitations. Notably, the insertion of an epitope tag can alter protein function. For a discussion of the effects of tag location on protein function, see Reference 8.

In addition to the traditional antibody and epitope combinations, other types of affinity tags have been discovered. An early example is the protein A tag, which binds to IgG (10). Other examples include those based on interaction with streptavidin (11) and biotin (12), maltosebinding peptide (MBP tag) and maltose (13), chitin-binding domain (CBD) and chitin (14), and the calmodulin-binding peptide that binds to calmodulin (15). Another type of tag is the S-peptide tag that binds to the S-protein derived from pancreatic RNase A (16).

Epitope tagging is accomplished by fusion of the target protein with the tag of choice. This is accomplished by insertion of the target gene into a host cell-specific expression vector that also encodes the epitope tag. Expression vectors for a variety of host cell types have been developed including Escherichia coli, yeast, insect, and mammalian cells. In many cases, the recognition sequence for a protease is included after the coding sequence of the epitope tag to allow for release of the epitope tag. In the case of the FLAG tag, the epitope includes the recognition sequence for the protease enterokinase. Another option is use of self-cleaving tags in which a self-cleaving intein fused to the CBD tag is used (14). Vectors are available that allow for $\mathrm{N}$ terminal and C-terminal tagging. The discovery of PCR greatly facilitated creation of tagged proteins by making it possible to fuse the desired epitope to the target by designing PCR primers encoding the epitope. A further breakthrough was achieved with the development of recombination-based cloning vectors $(17,18)$, which have made it unnecessary to create a new construct each time fusion to a new tag is desired.

In addition to tagging of protein expression vector constructs, genomic 
epitope tagging has been described (19). Jarvik et al. initially created an epitope tag in the form of a mini-exon flanked by splice donor and acceptor sites for insertion into the intron of a plasmid clone of a target gene. The tag is termed the CD tag after the "central dogma" of biology because the target gene is tagged initially, followed by the expressed mRNA, and finally, after translation, the expressed protein. The technique was later improved by the use of transposons for insertion into the target gene (20). Ross-Macdonald et al. (21) established a transposon system for Saccharomyces cerevisae in which a gene fusion to a reporter gene can be reduced in size by Cre-lox recombination to generate an epitope tag.

Perhaps the single most important application of epitope-tagging is coimmunoprecipitation. In this technique, a bait protein fused to an epitope tag is transfected into cells. Following lysis of the transfected cells, the expressed bait protein is immunoprecipitated with antitag antibody coupled to beads. Proteins that interact directly with the bait protein are co-immunoprecipated. Typically, the protein complex is then analyzed by SDS-PAGE. Or alternatively, the bait protein may be eluted from the anti-tag antibody prior to electrophoresis. A common variation of this method uses co-transfection of the bait and interacting proteins, each tagged with a different epitope tag. After co-immunopreciptiation using antibody to the epitopetagged bait protein, the interacting protein may be detected by Western blot analysis using antibody against the other epitope tag. The power of this technique has contributed greatly to our understanding of protein interaction networks. A few recent examples include the use of FLAGtagged COX-2 to identify interacting scaffolding proteins of ubiquitin ligase (22), the use of myc-tagged FKBP38 to co-immunopreciptiate presenelin 1 and 2 (23), and myc-tagged phytochromes B and $\mathrm{D}$ to identify additional phytochrome family members that form heterodimers in Arabidopsis (24).

A further advancement is the use of multiple epitope tags to increase the signal strength in detection applications. Examples of multiple epitope tags include multiple copies of the FLAG (25), His (26), c-myc (24,26), and HA tags (21). In addition to the use of multiple copies of the same epitope, a further refinement is the use of tandem copies of different tags. This makes it possible to combine the desired features of two or more tags. For example, FLAG and His dual tagging allows for detection of fusion proteins with anti-FLAG antibody and purification by metal affinity chromatography (27). In tandem affinity purification (TAP), an IgGbinding domain and calmodulin-binding peptide with an intervening tobacco etch virus (TEV) protease cleavage site are used (15). In the first stage, tagged proteins are purifed by protein A affinity. The bound fusion protein is released from protein A by cleavage with TEV protease. In the next stage, the eluted fusion protein is further purified by calmodulin affinity in the presence of calcium, and the bound proteins are released by incubation with EGTA. After two stages of purification, a high purity sample is obtained. Moreover, TAP can be followed by mass spectrometry in order to identify proteins that interact with a tagged bait protein. Recently, a shorter 4.6 kDa TAP tag has been described, the SF-TAP tag (28), in which two tandem copies of the Strep-tag II were combined with the FLAG tag.

\section{Where It's Going}

The post-genomic era and the emergence of the field of proteomics have further accelerated the development of applications for epitope tagging. Following the complete sequencing of the $S$. cerevisiae genome, Ho et al. (29) and Gavin et al. (30) reported the use of epitope tagging for genome scale mapping of protein-protein interaction. Ho et al. expressed 725 FLAG-tagged bait proteins (29). The expressed proteins were affinitypurified and protein complexes were resolved by SDS-PAGE. Proteins were excised from the gel, subjected to tryptic digestion, and analyzed by tandem mass spectrometry resulting in 35,000 protein identifications. Gavin et al. (30) inserted gene-specific cassettes containing the TAP tag (15) by homologous recombination at the $3^{\prime}$ ends of 1739 genes. Following tandem affinity purification and gel electrophoresis, proteins were analyzed by MALDI-TOF mass spectrometry.

Table 1. Common Epitope Tags

\begin{tabular}{|llll|}
\hline Name & Sequence & Detection & Purification \\
\hline FLAG & DYKDDDDK & M1,M2, M5 & Immunoaffinity \\
$6 \times$ His & HHHHHH & Anti-His & Metal affinity \\
HA & YPYDVPDYA & 12 CA5 & Immunoaffinity \\
C-myc & EQKLISEEDL & $9 E 10$ & Immunoaffinity \\
GST & 220 aa GST & Anti-GST & Glutathione \\
Protein A & IgG-binding domain & IgG & IgG \\
CD & 18 aa exon & 12 CA5 & Immunoaffinity \\
Strep-tag & WSAPQFEK & Strep-Tactin & Strep-Tactin \\
MBP & Maltose-binding protein & Anti-MBP & Maltose \\
CBD & Chitin-binding domain & Anti-CBD & Chitin \\
S-tag & S-peptide & Anti-S peptide & S-peptide \\
Avitag & GLNDIFEAQKIEWHE & Avidin & Avidin \\
CBP & CBP peptide & Anti-CBP & Calmodulin \\
TAP & Calmodulin- and IgG-binding domains & Anti-CBP & Calmodulin and IgG \\
SF-TAP & Strep Tag II and FLAG & Anti-FLAG & Strep-Tactin \\
GST, glutathione-S-transferase; CBP, calmodulin-binding peptide. & & 19 \\
\hline
\end{tabular}


Analysis of the proteins associated with 589 purified tagged proteins resulted in the identification of 16,830 proteins. In a further refinement of this approach, Graumann et al. (26) used tandem affinity purification in combination with multidimensional protein identification technology (MudPIT). MudPIT avoids the use of electrophoresis by using multidimensional capillary chromatography and ion trap mass spectrometry. The authors constructed a new TAP tag consisting of nine histidines and nine myc epitopes with two intervening precision protease cleavage sites. TAP-MudPIT analysis of 22 yeast strains expressing tagged proteins as bait identified 102 previously known and 279 potential physical interactions.

In addition to mass spectrometry, another effective technique for proteomic studies is the use of protein arrays. One of the challenges in this technique is the difficulty of binding proteins to the surface of the array. Recently, Ramachandran et al. (31) used an approach where epitopetagged gene constructs are expressed directly on the surface of the array to which an antibody to the tag is bound. When expressed, the tagged protein is captured by the antibody.

The number of protein interactions in the human cell has been estimated to be 375,000 (32). Epitope tagging has proven to be a powerful tool in elucidating this complex network. The combination of epitope tagging with the techniques of mass spectrometry and protein arrays represents a significant step in the development of new tools for proteomics. Further development of high-throughput and automated techniques is needed to accelerate the pace of future discovery.

\section{ACKNOWLEDGEMENTS}

The author thanks David Kehoe for advice and comments on the manuscript.

\section{COMPETING INTERESTS STATEMENT}

The author declares no competing interests.

\section{REFERENCES}

1. Hopp, T.P., K.S. Prickett, V.L. Price, R.T. Libby, C.J. March, D.P. Cerretti, D.L. Urdal, and P.J. Conlon. 1988. A short polypeptide marker sequence useful for recombinant protein identification and purification. Bio/Technology 6:1204-1210.

2. Hochuli, E., W. Bannwarth, H. Döbeli, R. Gentz, and D. Stuber. 1988. Genetic approach to facilitate purification of recombinant proteins with a novel metal chelate adsorbent. Bio/Technology 6:13211325.

3. Smith, D.B. and K.S. Johnson. 1988. Single-step purification of polypeptides expressed in Escherichia coli as fusions with glutathione-S-transferase. Gene 67:31-40.

4. Field, J., J. Nikawa, D. Broek, B. MacDonald, L. Rodgers, I.A. Wilson, R.A. Lerner, and M. Wigler. 1988. Purification of RAS-responsive adenylyl cyclase complex from Saccharomyces ceverisiae by use of an epitope addition method. Mol. Cell. Biol. 8:2159-2165.

5. Evan, G.I., G.K. Lewis, G. Ramsay, and J.M. Bishop. 1985. Isolation of monoclonal antibodies specific for human c-myc proto-oncogene product. Mol. Cell. Biol. 5:3610-3616.

6. Brizzard, B.L., R.G. Chubet, and D.L. Vizard. 1994. Immunoaffinity purification of FLAG epitope-tagged bacterial alkaline phosphatase using a novel monoclonal antibody and peptide elution. BioTechniques 16:730-735.

7. Kaufmann, M., P. Linder, A. Honegger, K. Blank, M. Tschopp, G. Capitani, A. Pluckthun, and M.G. Grutter. 2002. Crystal structure of the anti-His tag antibody 3D5 single-chain fragment complexed to its antigen. J. Mol. Biol. 318:135-147.

8. Jarvik, J.W. and C.A. Telmer. 1998. Epitope tagging. Annu. Rev. Genet. 32:601-618.

9. Tsien, R.Y. 1998. The green fluorescent protein. Annu. Rev. Biochem. 67:509-544.

10. Uhlén, M., B. Nilsson, B. Guss, M. Lindberg, S. Gatenbeck, and L. Philipson. 1983. Gene fusion vectors based on the gene for staphylococcal protein A. Gene 23:369-378

11. Schmidt, T.G.M. and A. Skerra. 2007. The Streptag system for one-step purification and high-affinity detection or capturing of proteins. Nat. Protocols $2: 1528-1535$

12. Tucker, J. and R. Grisshammer. 1996. Purification of a rat neurotensin receptor expressed in Escherichia coli. Biochem. J. 317:891-899.

13. di Guan, C., P. Li, P.D. Riggs, and H. Inouye. 1988. Vectors that facilitate the expression and purification of foreign peptides in Escherichia coli by fusion to maltose-binding protein. Gene 67:21-30.

14. Chong, S., F.B. Mersha, D.G. Comb, M.E. Scott, D. Landry, L.M. Vence, F.B. Perler, J. Benner, et al. 1997. Single-column purification of free recombinant proteins using self-cleavable affinity tag derived from a protein splicing element. Gene 192:271-281.

15. Rigaut, G., A. Shevchenko, B. Rutz, M. Wilm, M. Mann, and B. Seraphin. 1999. A generic protein purification method for protein complex characterization and proteome exploration. Nat. Biotechnol. 17:1030-1032

16. Hackbarth, J.S., S.H. Lee, X.W. Meng, B.T. Vroman, S.H. Kaufmann, and L.M. Karnitz. 2004. S-peptide epitope tagging for protein purification, expression monitoring, and localization in mammalian cells. BioTechniques 37:835-839.

17. Hartley, J.L., G.F. Temple, and M.A. Brasch. 2000. DNA cloning using in vitro site-specific recombination. Genome Res. 10:1788-1795.

18. Liu, Q., M.Z. Li, D. Leibham, D. Cortez, and S.J. Elledge. 1998. The univector plasmid-fusion system, a method for rapid construction of recombinant DNA without restriction enzymes. Curr. Biol. 8:13001309

19. Jarvik, J.W., S.A. Adler, C.A. Telmer, V. Subramaniam, and A.J. Lopez. 1996. CD-tagging: a new approach to gene and protein discovery and analysis. BioTechniques 20:896-904

20. Telmer, C.A., P.B. Berget, B. Ballou, R.F. Murphy, and J.W. Jarvik. 2002. Epitope tagging genomic DNA using a CD-tagging Tn10 minitransposon. BioTechniques 32:422-430.

21. Ross-Macdonald, P., A. Sheehan, G.S. Roeder, and M. Snyder. 1997. A multipurpose transposon system for analyzing protein production, localization, and function in Saccharomyces cerevisiae. Proc. Natl. Acad. Sci. USA 94:190-195.

22. Neuss, H., X. Huang, B.K.J. Hetfeld, R. Deva, P. Henklein, S. Nigam, J.W. Mall, W. Schwenk, and W. Dubiel. 2007. The ubiquitin- and proteasomedependent degradation of COX-2 is regulated by the COP9 signalosome and differentially influenced by coxibs. J. Mol. Med. 85:961-970.

23. Wang, H.Q., Y. Nakaya, Z. Du, T. Yamane, M. Shirane, T. Kudo, M. Takeda, K. Takebayashi, et al. 2005. Interaction of presenelins with FKBP38 promotes apoptosis by reducing mitochondrial $\mathrm{Bcl}-$ 2. Hum. Mol. Genet. 14:1889-1902.

24. Sharrock, R.A. and T. Clark. 2004 Heterodimerization of type II phytochromes in Arabidopsis. Proc. Natl. Acad. Sci. USA 101:1150011505.

25. Hernan, R., K. Heuermann, and B. Brizzard. 2000. Multiple epitope tagging of expressed proteins for enhanced detection. BioTechniques 28:789-793.

26. Graumann, J., L.A. Dunipace, J.H. Seol, W.H. McDonald, J.R. Yates III, B.J. Wold, and R.J. Deshaies. 2004. Applicability of tandem affinity purification MudPIT to pathway proteomics in yeast Mol. Cell. Proteomics 3:226-237.

27. DiCiommo, D.P., A. Duckett, I. Burcescu, R. Bremner, and B.L. Gallie. 2004. Retinoblastoma protein purification and transduction of retina and retinoblastoma cells using improved alphavirus vectors. Invest. Ophthalmol. Vis. Sci. 45:3320-3329.

28. Gloeckner, C.J., K. Boldt, A. Schumacher, R. Roepman, and M. Ueffing. 2007. A novel tandem affinity purification strategy for the efficient isolation of native protein complexes. Proteomics 7:42284234.

29. Ho, Y., A. Gruhler, A. Heilbut, G.D. Bader, L. Moore, S.L. Adams, A. Millar, P. Taylor, et al. 2002. Systematic identification of protein complexes in Saccharomyces cervisiae by mass spectrometry. Nature 415:180-183.

30. Gavin, A.C., M. Bosche, R. Krause, P. Grandi, M. Marzioch, A. Bauer, J. Schultz, J.M. Rick, et al. 2002. Functional organization of the yeast proteome by systematic analysis of protein complexes. Nature 415:141-147.

31. Ramachandran, N., E. Hainsworth, B. Bhullar, S. Eisenstein, B. Rosen, A.Y. Lau, J.C. Walter, and J. LaBaer. 2004. Self-assembling protein microarrays. Science 305:86-90.

32. Ramani, A.K., R.C. Bunescu, R.J. Mooney, and E.M. Marcottee. 2005. Consolidating the set of known human protein-protein interactions in preparation for large-scale mapping of the human interactome. Genome Biol. 6:R40.

Address correspondence to Bill Brizzard, Indiana University Research and Technology Corporation, 501 North Morton Street, Suite 204, Bloomington, IN 47404, USA.e-mail:bbrizzar@indiana.edu

To purchase reprints of this article, contact: Reprints@BioTechniques.com 